\title{
Segmentation-Driven Compound Document Coding Based on H.264/AVC-INTRA
}

\author{
Alexandre Zaghetto and Ricardo L. de Queiroz, Senior Member, IEEE
}

\begin{abstract}
In this paper, we explore H.264/AVC operating in intraframe mode to compress a mixed image, i.e., composed of text, graphics, and pictures. Even though mixed contents (compound) documents usually require the use of multiple compressors, we apply a single compressor for both text and pictures. For that, distortion is taken into account differently between text and picture regions. Our approach is to use a segmentation-driven adaptation strategy to change the H.264/AVC quantization parameter on a macroblock by macroblock basis, i.e., we deviate bits from pictorial regions to text in order to keep text edges sharp. We show results of a segmentation driven quantizer adaptation method applied to compress documents. Our reconstructed images have better text sharpness compared to straight unadapted coding, at negligible visual losses on pictorial regions. Our results also highlight the fact that H.264/AVC-INTRA outperforms coders such as JPEG-2000 as a single coder for compound images.
\end{abstract}

Index Terms-Compound document compression, document coding, H.264/AVC, mixed raster content.

\section{INTRODUCTION}

$\mathbf{T}$ HE newest video coding standard, the H.264/AVC [1], has been well explained in the literature [2]-[7]. Many papers have illustrated its performance showing many comparative results against coders such as MPEG-2. All results point to at least a factor of two improvement over previous standards. The reasons why the AVC is so good are many small improvements over previous methods. Each improvement brings a small coding gain, adding up to significant gains.

Apart from the factor-of-two improvement over other standards, there are a few unexpected advantages that come with the AVC package. One of these advantages is the intraframe macroblock prediction method, which will be explored in this paper.

\section{A. H.264/AVC-INTRA}

H.264/AVC is a video compression standard and it was not conceived to be applied as a still image compression tool. Nevertheless, the many coding advances brought into H.264/AVC, not only set a new benchmark for video compression, but they also make it a formidable compressor for still images [8], [9]. One of the components of these advances is the intraframe

Manuscript received December 4, 2006; revised March 13, 2007. The associate editor coordinating the review of this manuscript and approving it for publication was Dr. Giovanni Poggi.

The authors are with the Department of Electrical Engineering, Universidade de Brasilia, Brasilia, DF, 70910-900, Brazil (e-mail: zaghetto@image.unb.br; queiroz@ieee.org).

Color versions of one or more of the figures in this paper are available online at http://ieeexplore.ieee.org.

Digital Object Identifier 10.1109/TIP.2007.899036

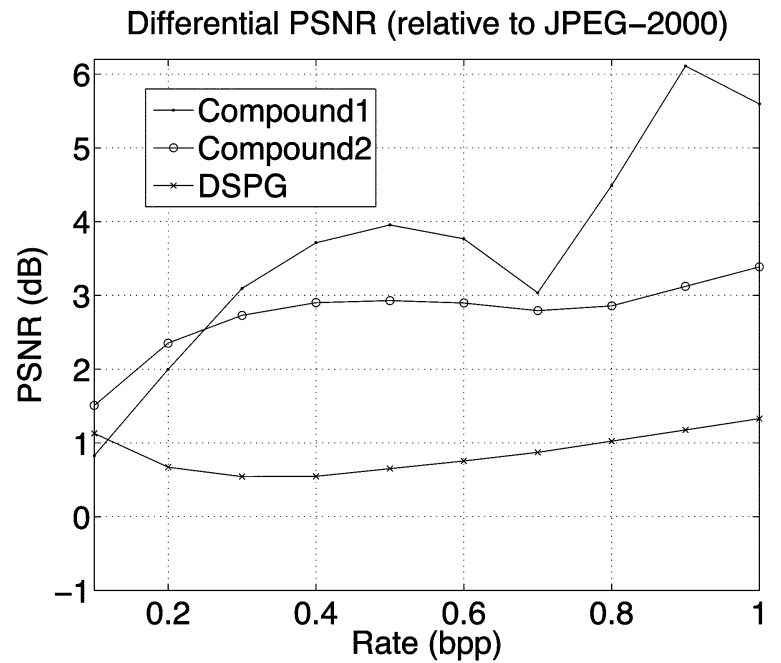

Fig. 1. Differential PSNR (relative to JPEG-2000) plots comparing AVC-I against JPEG-2000 for "compound1," "compound2," and "DSPG" images. PSNR gains surpass the mark of $3 \mathrm{~dB}$ in some cases. The compoundN images belong to the JPEG-2000 test set. Because of the very large size of "compound2," we selected only a portion of it for tests.

macroblock prediction method, which, combined with the context-adaptive binary arithmetic coding (CABAC), turns the H.264/AVC into a powerful still image compression engine. If we set our H.264/AVC implementation to work on a sole "INTRA" frame, it will behave as a still image compressor. We refer to this coder as AVC-I. The big surprise is that it also outperforms previous state-of-art coders, such as JPEG-2000 [10]. This is a surprise to many because it was not meant to be an image coder at all. However, results are consistent and unison.

Gains of the AVC-I over JPEG-2000 are typically in the order of 0.25 to $0.5 \mathrm{~dB}$ in PSNR for pictorial images [9], [11]. However, the AVC-I seems to have an extra capacity of adapting itself to heterodox content. For compound images (mixed pictures and text) the PSNR gains are more substantial, even surpassing the mark of 3-dB improvement in some cases, as shown in Fig. 1. Without a doubt, the AVC-I sets a new level of performance that will likely influence future still image coding standards.

\section{B. Compound Documents}

Electronic documents are basically represented in two forms: vectorial or raster [12]. It is not much of a challenge to compress vectorized documents since each object can be compressed individually and the whole file can be compressed losslessly. The real challenge is to compress rasterized documents. Compound documents are assumed here as raster documents which contain a mix of text and pictorial contents. 


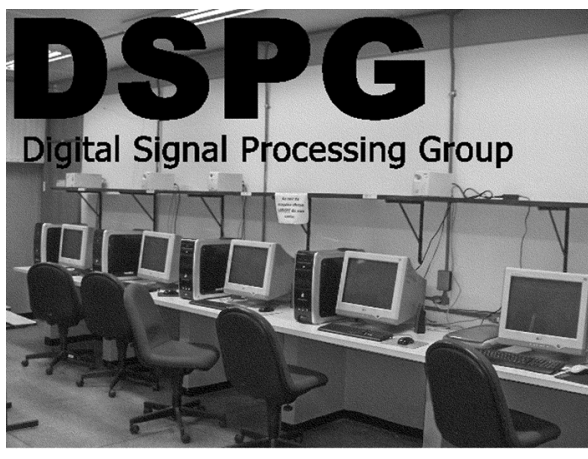

(a)

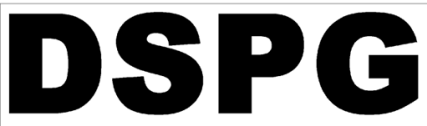

Digital Signal Processing Group

(b)

Fig. 2. Text segmentation algorithm: (a) original grayscale image $(2592 \times 1952$ pels $)$; (b) its segmented text (binary).

Compression algorithms are developed with a particular image type, characteristic and application in mind and no single algorithm is best across all types of images or applications. When compressing text, it is important to preserve the edges and shapes of characters accurately to facilitate reading. The human visual system, however, works differently for typical continuous-tone images, better masking high-frequency errors [13].

Compound raster documents have typically been compressed as a single image. However, different compression algorithms may be applied to each of the regions of the document. That is the way multiple-coder-based algorithms work [12]. Instead of a multiple-code approach, this paper proposes a single-coder algorithm based on a modified version of the AVC-I that adjusts itself as an effort to encode text and pictorial regions differently.

\section{Motivation and Context}

It is important to place our coder within the proper application context. We are concerned with a distributed scenario such as the web, where a document is stored once, somewhere, and many users are to retrieve and decode the document at a later time. In this scenario, the encoder has no complexity restriction. Indeed, as we will see later, we may use a large number of passes at encoding time and we apply segmentation algorithms, as well. These high-complexity operations are to be done off-line and do not affect the decoding process at all. Thus, our encoder complexity was not considered here in comparison to other encoders such as JPEG-2000. We assume it is important to have standard decoders that might already be available to normal users. The AVC-I is not a still image coding standard, but since it is based

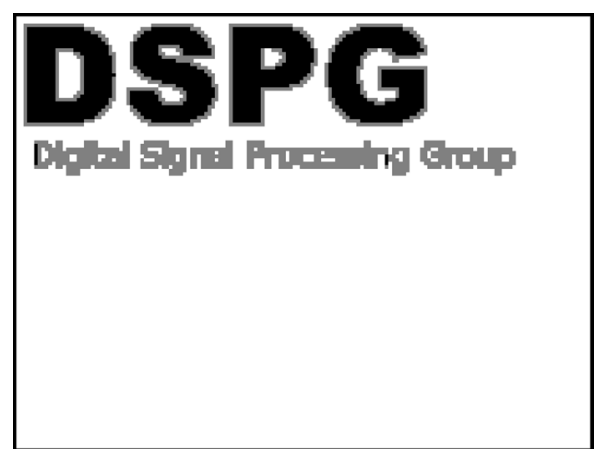

(a)

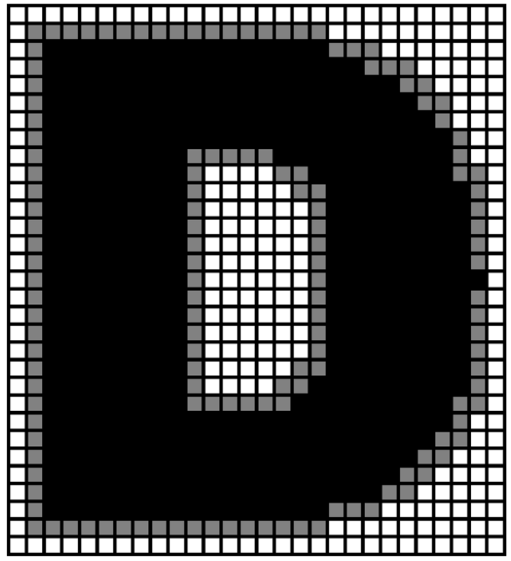

(b)

Fig. 3. Coding mask: (a) macroblocks that belong to classes 0,1 , and 2 are labeled as 255 (white), 0 (black) and 128 (gray), respectively; (b) zoom around character "D" in "DSPG." The grid shows the $16 \times 16$ pixels macroblocks.

on a video standard that outperforms the state of the art in still image coding, we are looking into the future and forecasting a new standard based on AVC-C standard or on similar techniques.

\section{Segmentation-Driven Rate Allocation}

A few authors dealt with compressing documents with a single coder. For example, Konstantinides and Tretter [14] used adaptive quantization within the JPEG extensions framework to compress compound (mixed) images. The idea is to use less aggressive quantizer steps for text regions in order to keep edges sharp, while being more forgiving to high frequency losses in pictures. Ramos and De Queiroz [15] used a single JPEG coder for the compression of mixed documents, stealing bits from background and images to give to text and sharp graphics edges. For that, thresholding was used, which is a technique that analyzes each quantized DCT coefficients in JPEG, computing how many bits it costs to be transmitted and how much distortion it would cause to the reconstructed image if it is not transmitted at all. In effect, thresholding is an optimization technique for JPEG based on the rate-distortion (RD) analysis of every block. Coefficients which are not rate-distortion efficient are discarded (thresholded). It was just a case of making the $\mathrm{RD}$ analysis adaptive depending on the image contents.

In general, for RD-optimized transform coding, the signal is divided into units $x_{i}$, each contributing to the overall bit-rate $R$ 


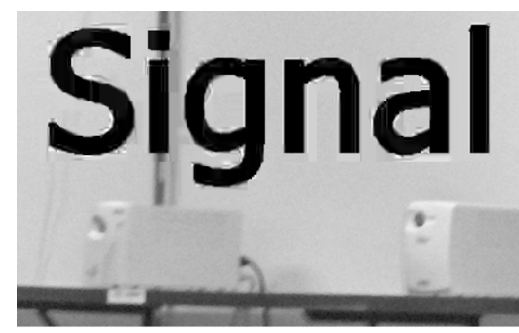

(a)

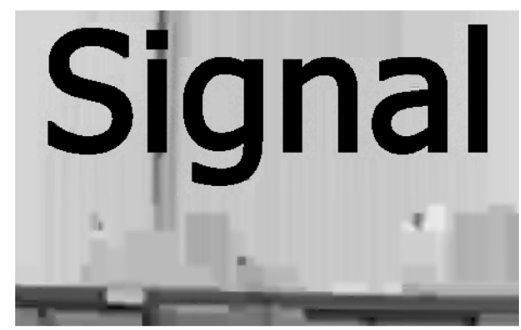

(b)

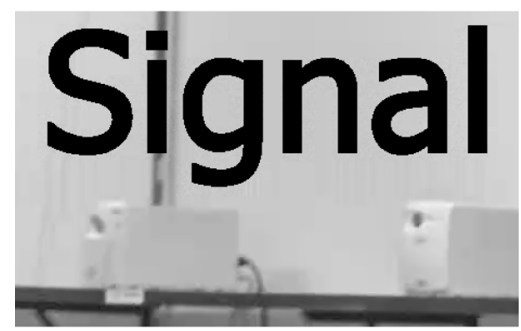

(c)

Fig. 4. AVC-C Tests. (a) $Q_{p}=0, Q_{p \text { Text }}=51$ : best quality for class 0 and 1 (pictorial and text interior) MBs, worst quality for class 2 (text border) MBs; (b) $Q_{p}=51, Q_{p \text { Text }}=0$ : best quality for class 2 (text border) MBs, worst quality for class 0 and 1 (pictorial and text interior) MBs; (c) $Q_{p}=34$, $Q_{p \text { Text }}=17:$ proposed solution.

by $R_{i}$ bits, i.e., $R=\sum_{i} R_{i}$. Distortion is some function of the quantization error $\hat{x}_{i}-x_{i}$, where $\hat{x}_{i}$ is the reconstructed unit. The global distortion is given by

$$
D=f\left(\left\{\hat{x}_{i}-x_{i}, \forall i\right\}\right) \quad\left(\text { e.g. }=\sum_{i}\left[\hat{x}_{i}-x_{i}\right]^{2}\right)
$$

By using a well-behaved distortion function, such as MSE, then $D=\sum_{i} D_{i}$ where $D_{i}$ is the distortion for the $i$ th unit as $D_{i}=$ $\left[\hat{x}_{i}-x_{i}\right]^{2}$. RD optimization involves the minimization of a cost function $J=R+\lambda D$, where $\lambda$ is a Lagrangian multiplier. Hence

$$
J=\sum_{i} R_{i}+\lambda\left[\hat{x}_{i}-x_{i}\right]^{2}
$$

We imply a space varying meaning for distortion as opposed to adapting the algorithm, i.e., $D_{i}=\left[\hat{x}_{i}-x_{i}\right]^{2} u_{i}$, where $u_{i}$ is a distortion weighting factor specific for the $i$ th unit. In conventional human visual system (HVS) weighted error measures, we can use a frequency-based weighting system in the transform domain. Since the HVS response is not completely understood and cannot be easily modeled, one can classify the image blocks into a discrete number of representative classes and devise HVS weights for each of the classes. For simplicity we assign weights

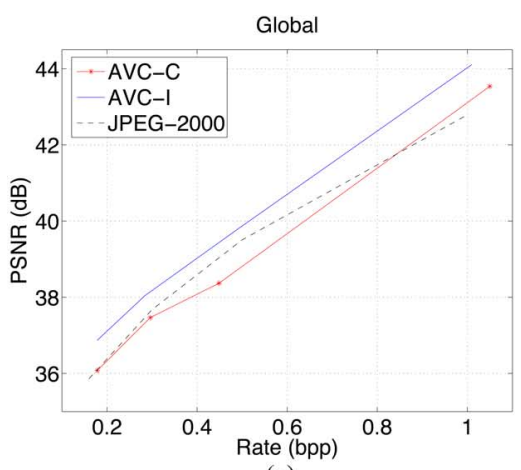

(a)

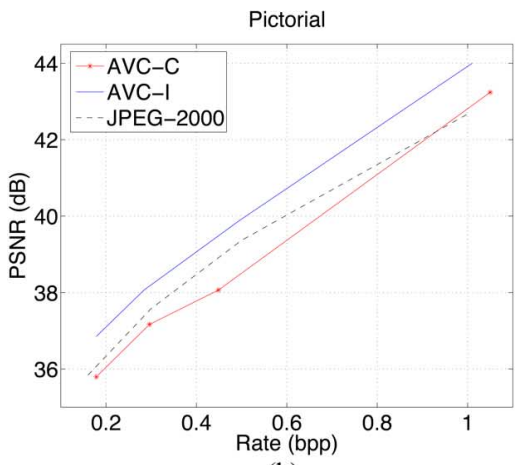

(b)

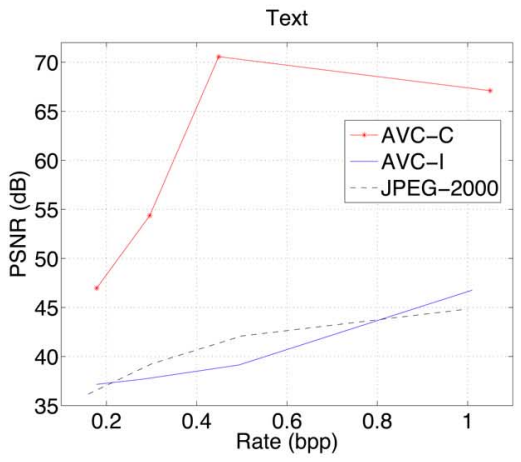

(c)

Fig. 5. Objective performance comparison between AVC-C, AVC-I and JPEG2000 for "DSPG" image: (a) global PSNR; (b) pictorial regions PSNR; (c) text regions PSNR. Notice that text regions quality can be considerably improved with little global quality loss.

$u_{i}$ for the error rather than weights in the transform domain. Hence

$$
J=\sum_{i} R_{i}+\lambda_{i}\left[\hat{x}_{i}-x_{i}\right]^{2}
$$

where $\lambda_{i}=u_{i} \lambda$.

In H.264/AVC, there are many decisions per block or macroblock that the encoder has to make. Examples are the division of the macroblock for motion estimation, the transform to be used $(4 \times 4$ or $8 \times 8)$, the intraframe block prediction mode, etc. These decisions are all made by rate-distortion considerations. Apart from that, we can always use thresholding based on RD too, just like in JPEG. In all these cases, we have to make an RD analysis per macroblock.

H.264/AVC allows for the change of the quantizer parameter $Q_{p}$ at each macroblock. The adjustment of $\lambda$, or $\lambda_{i}$, in the quantization step, is translated into an adjustment of $Q_{p}$ by an expo- 
TABLE I

AVC-C AdJUSTED WITH $Q_{p}=21$ AND $Q_{p \text { Text }}=03$. AVC-I AdJUSTED WITH $Q_{p}=20$. JPEG-2000 ADJUSTED WITH bitrate $=1.00 \mathrm{bpp}$

\begin{tabular}{|c|c|c|c|c|}
\hline $\begin{array}{c}\text { Result/ } \\
\text { Algorithm }\end{array}$ & $\begin{array}{c}\text { PSNR } \\
\text { Global } \\
(\mathrm{dB})\end{array}$ & $\begin{array}{c}\text { PSNR } \\
\text { Pictorial } \\
(\mathrm{dB})\end{array}$ & $\begin{array}{c}\text { PSNR } \\
\text { Textual } \\
(\mathrm{dB})\end{array}$ & $\begin{array}{c}\text { Bitrate } \\
(\mathrm{bpp})\end{array}$ \\
\hline AVC-C & 43.54 & 43.24 & 67.10 & 1.05 \\
\hline AVC-I & 44.11 & 44.00 & 46.76 & 1.01 \\
\hline JPEG-2000 & 42.79 & 42.67 & 44.84 & 1.00 \\
\hline
\end{tabular}

TABLE II

AVC-C: $Q_{p}=28$ AND $Q_{p \text { Text }}=02$. AVC-I: $Q_{p}=25$ JPEG-2000: bitrate $=0.50 \mathrm{bpp}$

\begin{tabular}{|c|c|c|c|c|}
\hline $\begin{array}{c}\text { Result/ } \\
\text { Algorithm }\end{array}$ & $\begin{array}{c}\text { PSNR } \\
\text { Global } \\
(\mathrm{dB})\end{array}$ & $\begin{array}{c}\text { PSNR } \\
\text { Pictorial } \\
(\mathrm{dB})\end{array}$ & $\begin{array}{c}\text { PSNR } \\
\text { Textual } \\
(\mathrm{dB})\end{array}$ & $\begin{array}{c}\text { Bitrate } \\
(\mathrm{bpp})\end{array}$ \\
\hline AVC-C & 38.37 & 38.07 & 70.56 & 0.45 \\
\hline AVC-I & 39.81 & 39.87 & 39.13 & 0.49 \\
\hline JPEG-2000 & 39.51 & 39.37 & 42.09 & 0.50 \\
\hline
\end{tabular}

TABLE III

AVC-C: $Q_{p}=30$ AND $Q_{p \text { Text }}=11$. AVC-I: $Q_{p}=28$. JPEG-2000: bitrate $=0.30 \mathrm{bpp}$

\begin{tabular}{|c|c|c|c|c|}
\hline $\begin{array}{c}\text { Result/ } \\
\text { Algorithm }\end{array}$ & $\begin{array}{c}\text { PSNR } \\
\text { Global } \\
(\mathrm{dB})\end{array}$ & $\begin{array}{c}\text { PSNR } \\
\text { Pictorial } \\
(\mathrm{dB})\end{array}$ & $\begin{array}{c}\text { PSNR } \\
\text { Textual } \\
(\mathrm{dB})\end{array}$ & $\begin{array}{c}\text { Bitrate } \\
(\mathrm{bpp})\end{array}$ \\
\hline AVC-C & 37.46 & 37.17 & 54.36 & 0.30 \\
\hline AVC-I & 38.04 & 38.07 & 37.71 & 0.28 \\
\hline JPEG-2000 & 37.67 & 37.58 & 39.24 & 0.30 \\
\hline
\end{tabular}

TABLE IV

AVC-C: $Q_{p}=34$ AND $Q_{p \text { Text }}=17$. AVC-I: $Q_{p}=31$. JPEG-2000: bitrate $=0.16 \mathrm{bpp}$

\begin{tabular}{|c|c|c|c|c|}
\hline $\begin{array}{c}\text { Result/ } \\
\text { Algorithm }\end{array}$ & $\begin{array}{c}\text { PSNR } \\
\text { Global } \\
(\mathrm{dB})\end{array}$ & $\begin{array}{c}\text { PSNR } \\
\text { Pictorial } \\
(\mathrm{dB})\end{array}$ & $\begin{array}{c}\text { PSNR } \\
\text { Textual } \\
(\mathrm{dB})\end{array}$ & $\begin{array}{c}\text { Bitrate } \\
(\mathrm{bpp})\end{array}$ \\
\hline AVC-C & 36.08 & 35.80 & 46.96 & 0.17 \\
\hline AVC-I & 36.87 & 36.85 & 37.16 & 0.18 \\
\hline JPEG-2000 & 35.86 & 35.84 & 36.17 & 0.16 \\
\hline
\end{tabular}

nential equation. The quantizer adjustment is the most effective way to control rate and distortion. It controls more intensively the RD balance than for example using RD analysis to select the best macroblock prediction mode, or the size of the DCT. Therefore, we can cut corners and adjust RD (minimize $J$ ) by modifying directly the quantizer parameter at each macroblock.

We propose to adapt the analysis on a macroblock by macroblock basis to be more economic in some blocks as opposed to others. First, we apply a region classification algorithm that will identify text and pictorial regions. This classification algorithm is derived from an edge detector and needs to identify edges belonging to text as opposed to textures. We assume that, in these text regions, the viewer would pay greater attention to edges.

In segmentation-driven image compression techniques, the image to be compressed is first segmented [16]. Since segmentation is not the main focus of this paper, we used Fan's text segmentation algorithm [17] in our tests for convenience. Any other text segmentation algorithm could be used, as well. As an example, Fig. 2(a) shows a compound document which is input to the segmenter. The output is shown in Fig. 2(b).

The next step is to classify [18] each macroblock $(16 \times 16$ pixels block), denoted here as MB. The binary image containing

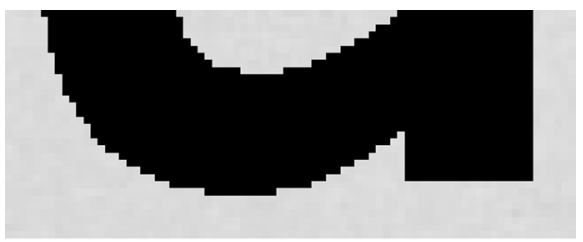

(a)

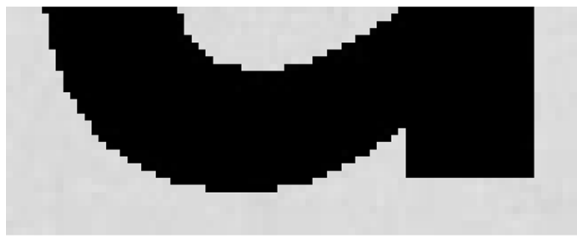

(b)

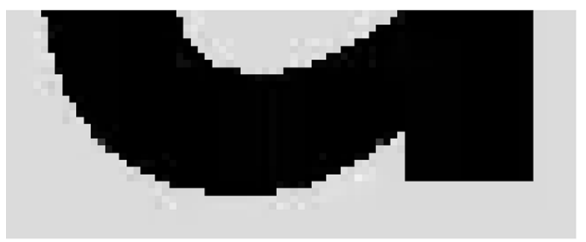

(c)

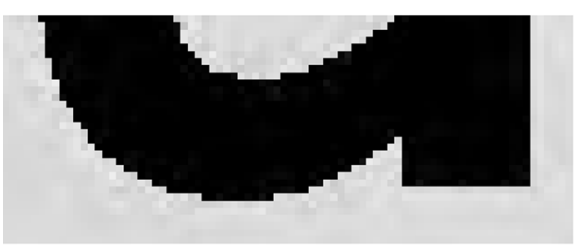

(d)

Fig. 6. Subjective performance comparison between AVC-C, AVC-I and JPEG-2000 in text regions. Zoom of lower part of character "u" in the word "Group" of "DSPG" image: (a) original; (b) AVC-C encoded; (c) AVC-I encoded; (d) JPEG-2000 encoded. Bitrate is 0.16 bpp.

the segmented text is analyzed and each MB is classified as type 0,1 , or 2 and a coding mask is constructed. MBs of class 0 (pictorial regions) are composed exclusively by pixels marked as background. Class $1 \mathrm{MBs}$ (text interior regions) are those composed exclusively by pixels marked as text. MBs which present a mixture of background and text interior, in any proportion, are considered as class 2 (text border macroblock). Fig. 3(a) shows the coding mask of Fig. 2(b). Fig. 3(b) shows an enlarged portion of "D" in "DSPG." To make it easier to visualize, MB classes 0,1 , and 2 were represented as white, black and gray, respectively.

The coding mask is passed on to a modified version of AVC-I, which will adapt the value of $Q_{p}$ for each $\mathrm{MB}$, according to the class it belongs. The idea is to "transfer" quality of a MB class to another. Class 0 and 1 regions are encoded with a quantizer parameter $Q_{p}$, while class 2 regions are encoded with a quantizer parameter $Q_{p \text { Text }}$, being $Q_{p \text { Text }}<Q_{p}$. This means that more compression is applied where there is texture, and less compression is applied to the text letter borders. This algorithm is referred to as H.264/AVC-INTRA Compound, or simply AVC-C.

Alternatively, it can be used to encode a region of interest (ROI) differently from the rest. The idea is to allow a single H.264 coder to compress more efficiently compound images. 


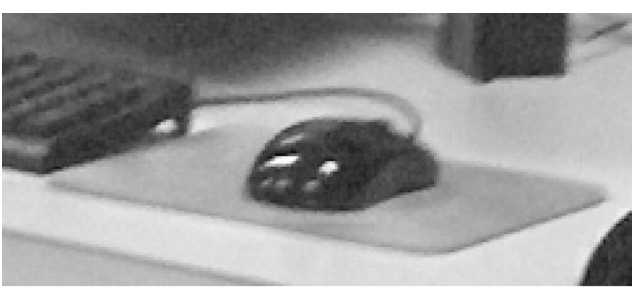

(a)

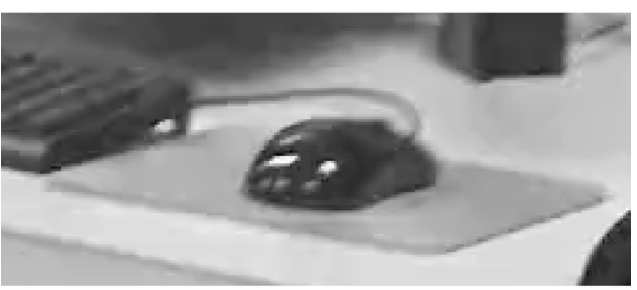

(b)

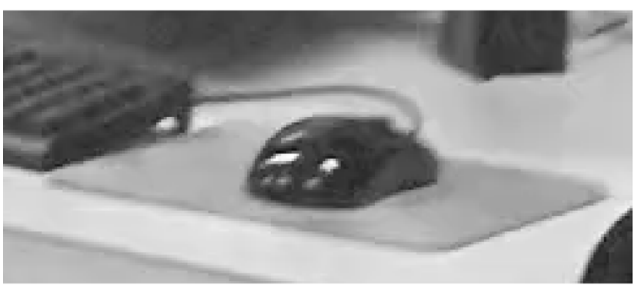

(c)

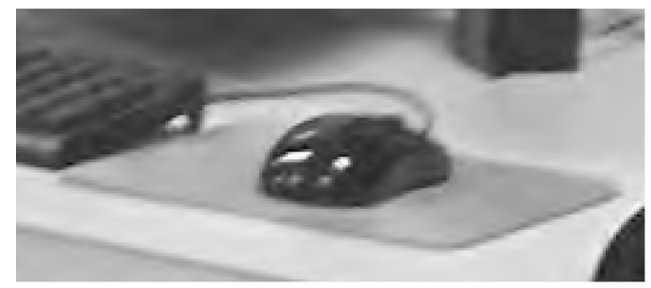

(d)

Fig. 7. Subjective performance comparison between AVC-C, AVC-I and JPEG-2000 for pictorial regions. (a) original; (b) AVC-C encoded; (c) AVC-I encoded; (d) JPEG-2000 encoded. Bitrate is 0.16 bpp.

\section{Text Versus Picture Balance}

Some tests were carried to verify the performance of the proposed encoder. Fig. 4 shows results of AVC-C adjusted with (a) $Q_{p}=0$ and $Q_{p \text { Text }}=51$ (best quality for classes 0 and 1 and worst quality for class 2) and (b) $Q_{p}=51$ and $Q_{p \text { Text }}=0$ (best quality for class 2 and worst quality for classes 0 and 1). Although, in Fig. 4(b), text border regions are sharp, this is not enough. It is also desired to maintain the quality of pictorial regions at reasonable levels. In other words, we want to lower the quality of pictorial and text interior regions to improve text border regions until they become sufficiently clear, without compromising the quality of the whole document. The results presented in Fig. 4 only show that the encoder is capable to adapt itself based on context, as foreseen.

Our $Q_{p}$ and $Q_{p \text { Text }}$ selection algorithm works as follows.

1) A bitrate $R$ is chosen.

2) A bitrate variation $\delta r$ around $R$ is set.

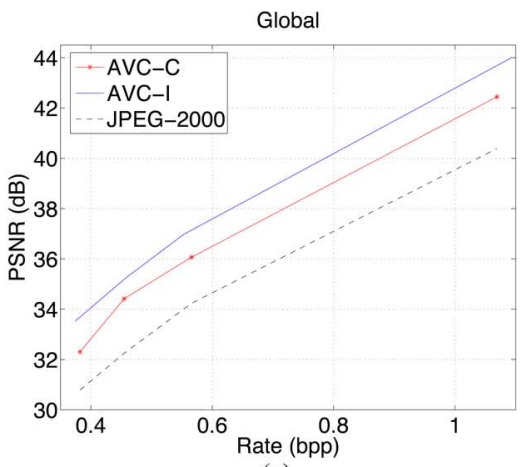

(a)

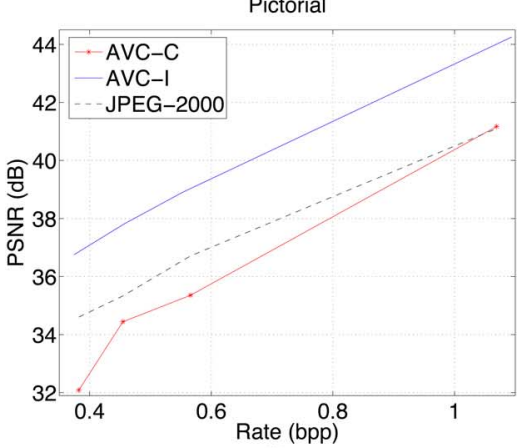

(b)

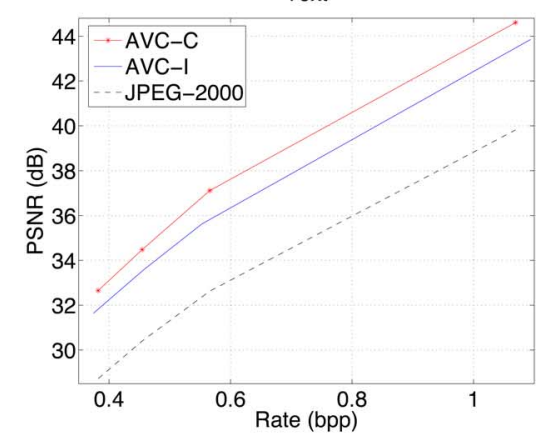

(c)

Fig. 8. Objective performance comparison between AVC-C, AVC-I and JPEG-2000 for part of "Compound2" image: (a) global PSNR; (b) pictorial regions PSNR; (c) text regions PSNR. Notice that text regions quality can be considerably improved with little global quality loss.

3) Among all possible $\left(Q_{p}, Q_{p \text { Text }}\right)$ combinations, those which present bitrates inside the interval $R \pm \delta r$ are selected.

4) Among all selected combinations, the maximum PSNR value, PSNRmax, is determined.

5) A PSNR variation $\delta q$ is set, and a minimum PSNR value, PSNRmin $=$ PSNRmax $-\delta q$, is calculated.

6) Among all selected $\left(Q_{p}, Q_{p \text { Text }}\right)$ in step 3$)$, those whose PSNR values are greater than PSNRmin are chosen as candidates.

7) The candidate with the largest $d=Q_{p}-Q_{p \text { Text }}$ (better quality for text border regions) is picked.

Since complexity is not an issue, as previously discussed, AVC-C computes PSNR and bitrate values for all $\left(Q_{p}, Q_{p \text { Text }}\right)$ combinations per document, before running the selection algorithm. 
The image shown in Fig. 2(a) was compressed by AVC-C (adaptive $Q_{p}$ ), AVC-I (fixed $Q_{p}$ ) and JPEG-2000 (fixed bitrate) with different configurations, and results are shown in Tables I-IV. The results were also used to plot the graphics shown in Fig. 5.

The differences among the coders become more clear when text is visualized in details. Fig. 6 shows the zoomed region of a text letter border for (a) the original image and its reconstructed (b) AVC-C, (c) AVC-I, and (d) JPEG-2000 versions, after compression at $0.16 \mathrm{bpp}$. Enlarged portions of the pictorial region of the same images are shown in Fig. 7.

Fig. 8 shows the PSNR plots for part of "Compound2" image.

\section{CONCLUSIONS}

The AVC-I is very effective for compound documents because of its intraframe prediction mode. With AVC-C, for the same bitrate, it is possible to improve significantly the quality of text regions, with little to negligible losses to the pictorial regions. The losses to pictorial regions yield an overall PSNR loss so small that AVC-C still outperforms JPEG-2000. Even though there is not an overall objective gain over AVC-I, the proposed AVC-C encodes text regions at higher quality and yields an overall higher quality to the image. Furthermore, the proposed AVC-C encoder is compatible with AVC-I decoder.

Future works might consider extending the number of classes.

\section{REFERENCES}

[1] Final Draft International Standard of Joint Video Specification, ITU-T Recommendation H.264, ISO/IEC 14496-10 AVC,, Mar. 2003.

[2] I. E. G. Richardson, H.264 and MPEG-4 Video Compression. New York: Wiley, 2003.

[3] T. Wiegand, G. J. Sullivan, G. Bjontegaard, and A. Luthra, "Overview of the H.264/AVC video coding standard," IEEE Trans. Circuits Syst. Video Technol., vol. 13, no. 7, pp. 560-576, Jul. 2003.

[4] T. Stockhammer, M. M. Hannuksela, and T. Wiegand, "H.264/AVC in wireless environments," IEEE Trans. Circuits Syst. Video Technol., vol. 13, no. 7, pp. 657-673, Jul. 2003.

[5] T. Wiegand, H. Schwarz, A. Joch, F. Kossentini, and G. J. Sullivan, "Rate-constrained coder control and comparison of video coding standards," IEEE Trans. Circuits Syst. Video Technol., vol. 13, no. 7, pp. 688-703, Jul. 2003.

[6] G. J. Sullivan, P. Topiwala, and A. Luthra, "The H.264/AVC advanced video coding standard: Overview and introduction to the fidelity range extensions," presented at the 27th SPIE Conf. Applications Digital Image Processing, Aug. 2004.

[7] J. Ostermann, J. Bormans, P. List, D. Marpe, M. Narroschke, F. Pereira, T. Stockhammer, and T. Wedi, "Video coding with H.264/AVC: Tools, performance, and complexity," IEEE Circuits Syst. Mag., vol. 4, no. 1, pp. 7-28, Mar. 2004.

[8] D. Marpe, V. George, and T. Wiegand, "Performance comparison of intra-only H.264/AVC and JPEG2000 for a set of monochrome ISO/IEC test images," Contribution JVT ISO/IEC MPEG and ITU-T VCEG, JVT M-014, Oct. 2004.

[9] D. Marpe, V. George, H. L. Cycon, and K. U. Barthel, "Performance evaluation of Motion-JPEG2000 in comparison with H.264/AVC operated in pure intra coding mode," Proc. SPIE, vol. 5266, pp. 129-137, 2004 , Wavelet applications in industrial processing.
[10] D. S. Taubman and M. W. Marcellin, JPEG2000: Image Compression Fundamentals, Standards, and Practice. Norwell, MA: Kluwer, 2002.

[11] R. L. de Queiroz, R. S. Ortis, A. Zaghetto, and T. A. Fonseca, "Fringe benefits of the H.264/AVC," in Proc. Int. Telecom. Symp., Fortaleza, Brazil, Sep. 2006, pp. 208-212.

[12] R. L. de Queiroz, "Compressing compound documents," in The Document and Image Compression Handbook, M. Barni, Ed. New York: Marcel-Dekker, 2005.

[13] D. Marr, Vision. San Francisco, CA: Freeman, 1982.

[14] K. Konstantinide and D. Tretter, "A JPEG variable quantization method for compound documents," IEEE Trans. Image Process., vol. 9, no. 7, pp. 1282-1287, Jul. 2000.

[15] M. Ramos and R. de Queiroz, "Classified JPEG coding of mixed documents," IEEE Trans. Image Process., vol. 9, no. 4, pp. 716-720, Apr. 2000.

[16] M. Kunt, A. Ikonomopoulos, and M. Kocher, "Second-generation image-coding techniques," Proc. IEEE, vol. 73, no. 4, pp. 549-574, Apr. 1985.

[17] J. Fan, "Text extraction via an edge-bounded averaging and a parametric character model," in Proc. SPIE Document Recognition and Retrieval X, Santa Clara, CA, Jan. 2003, vol. 5010, pp. 8-19.

[18] B. Ramamurthi and A. Gersho, "Classified vector quantization of images," IEEE Trans. Commun., vol. 34, no. 11, pp. 1105-1115, Nov. 1986.

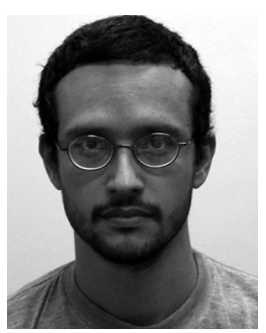

Alexandre Zaghetto received the Engineer degree from the Federal University of Rio de Janeiro, Rio de Janeiro, Brazil, in 2002, and the M.Sc. degree from the University of Brasilia, Brasilia, Brazil, in 2004, both in electrical engineering. He is currently pursuing the Ph.D. degree at the Department of Electrical Engineering, University of Brasilia.

His main research interests are in image processing and compound document coding.

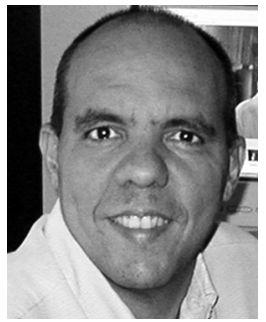

Ricardo L. de Queiroz (S'86-M'94-SM'99) received the Engineer degree from the Universidade de Brasilia, Brasilia, Brazil, in 1987, the M.Sc. degree from Universidade Estadual de Campinas, Brazil, in 1990, and the Ph.D. degree from The University of Texas at Arlington, in 1994, all in electrical engineering.

From 1990 to 1991, he was with the DSP research group at Universidade de Brasilia, as a Research Associate. He joined Xerox Corporation in 1994, where he was a member of the research staff until 2002. From 2000 to 2001, he was also an Adjunct Faculty at the Rochester Institute of Technology, Rochester, NY. He is now with the Electrical Engineering Department, Universidade de Brasilia. He has published extensively in journals and conferences and contributed chapters to books, as well. He also holds 36 issued patents. His research interests include image and video compression, multirate signal processing, and color imaging.

Dr. de Queiroz is a member of the IEEE Signal Processing Society Image and MultiDimensional Signal Processing (IMDSP) Technical Committee. He is an Associate Editor for the IEEE TRANSACTIONS ON IMAGE PROCESSING, the IEEE TRANSACTIONS ON CIRCUITS AND SySTEMS FOR VIDEO TECHNOLOGY, and a past Associate Editor for the IEEE SignAl PROCESSING LETTERS. He has been actively involved with the Rochester chapter of the IEEE Signal Processing Society, where he served as Chair and organized the Western New York Image Processing Workshop since its inception until 2001. He was also part of the organizing committee of ICIP 2002. He is a member of the Brazilian Telecommunications Society. 